JOURNAL OF THE

AMERICAN MATHEMATICAL SOCIETY

Volume 16, Number 1, Pages 19-28

S 0894-0347(02)00401-0

Article electronically published on July 10, 2002

\title{
THE PLANAR CANTOR SETS OF ZERO ANALYTIC CAPACITY AND THE LOCAL $T(b)$-THEOREM
}

\author{
JOAN MATEU, XAVIER TOLSA, AND JOAN VERDERA
}

\section{INTRODUCTION}

In this paper we characterize the planar Cantor sets of zero analytic capacity. Our main result answers a question of P. Mattila $\mathrm{Ma}$ and completes the solution of a long-standing open problem with a curious history, which goes back to 1972 . We refer the reader to [I2, p. 153] and [Ma for more details. Moreover, we confirm a conjecture of Eiderman $[\mathrm{E}]$ concerning the analytic capacity of the $N$-th approximation of a Cantor set.

Before formulating our main results, we recall the definition of the basic objects involved.

The analytic capacity of a compact subset $E$ of the complex plane $\mathbb{C}$ is

$$
\gamma(E)=\sup \left|f^{\prime}(\infty)\right|,
$$

where the supremum is taken over all analytic functions $f$ on $\mathbb{C} \backslash E$ such that $|f| \leqslant 1$ on $\mathbb{C} \backslash E$. Although there has recently been important progress on our understanding of analytic capacity (see the survey papers $[\mathrm{D}, \mathrm{V3}$ and the references given there), many basic questions about $\gamma$ remain unanswered. One of the oldest is the semiadditivity problem, that is, the problem of showing the existence of an absolute constant $C$ such that

$$
\gamma(E \cup F) \leqslant C\{\gamma(E)+\gamma(F)\},
$$

for all compact sets $E$ and $F$. If (2) were true, then one would have powerful geometric criteria for rational approximation, which are otherwise missing (see $\mathrm{V} 2$ and $[\mathrm{Vi2}])$.

On the other hand, it has recently been established that a close variant of $\gamma$, called positive analytic capacity, is indeed semi-additive. The positive analytic capacity of a compact set $E$ is

$$
\gamma^{+}(E)=\sup \mu(E)
$$

Received by the editors August 7, 2001.

2000 Mathematics Subject Classification. Primary 30C85; Secondary 42B20, 30E20.

Key words and phrases. Analytic capacity, Cauchy integral, Cantor sets, T(b)-Theorem, positive analytic capacity.

The authors were partially supported by the grants BFM 2000-0361, HPRN-2000-0116 and 2001- SGR-00431. The second author was supported by a Marie Curie Fellowship of the European Union under contract HPMFCT-2000-00519.

(C)2002 American Mathematical Society 
where the supremum is taken over the positive Borel measures $\mu$ supported on $E$ such that the Cauchy potential $f=\frac{1}{z} * \mu$ is a function in $L^{\infty}(\mathbb{C})$ with $\|f\|_{\infty} \leqslant 1$. Since $\left(\frac{1}{z} * \mu\right)^{\prime}(\infty)=\mu(E)$, we clearly have $\gamma^{+}(E) \leqslant \gamma(E)$.

Since $\gamma^{+}$is semi-additive, as shown in [T1], it is clear that (21) follows from the inequality

$$
\gamma(E) \leqslant C \gamma^{+}(E), \quad E \text { compact } \subset \mathbb{C}
$$

where the positive constant $C$ does not depend on $E$. We will see below that our main result provides a proof of (3) for a particular (but significant) class of sets $E$. To the best of our knowledge, the first mention of (3) that can be found in the literature is in DO. An equivalent form of (3), which involves Menger curvature, has recently been conjectured by Melnikov (see [D]).

Now we turn our attention to Cantor sets. Given a sequence $\lambda=\left(\lambda_{n}\right)_{n=1}^{\infty}$, $0 \leqslant \lambda_{n} \leqslant 1 / 3$, we construct a Cantor set by the following algorithm. Consider the unit square $Q^{0}=[0,1] \times[0,1]$. At the first step we take four closed squares inside $Q^{0}$, of side-length $\lambda_{1}$, with sides parallel to the coordinate axes, such that each square contains a vertex of $Q^{0}$. At step 2 we apply the preceding procedure to each of the four squares produced at step 1 , but now using the proportion factor $\lambda_{2}$. Then we obtain 16 squares of side-length $\sigma_{2}=\lambda_{1} \lambda_{2}$. Proceeding inductively, we have at the $n$-th step $4^{n}$ squares $Q_{j}^{n}, 1 \leqslant j \leqslant 4^{n}$, of side-length $\sigma_{n}=\prod_{j=1}^{n} \lambda_{j}$. Write

$$
E_{n}=E\left(\lambda_{1}, \ldots, \lambda_{n}\right)=\bigcup_{j=1}^{4^{n}} Q_{j}^{n},
$$

and define the Cantor set associated with the sequence $\lambda=\left(\lambda_{n}\right)_{n=1}^{\infty}$ by the identity

$$
E=E(\lambda)=\bigcap_{n=1}^{\infty} E_{n} .
$$

Our main result reads as follows.

Theorem 1. The Cantor set $E(\lambda)$ has zero analytic capacity if and only if

$$
\sum_{n=1}^{\infty} \frac{1}{\left(4^{n} \sigma_{n}\right)^{2}}=\infty
$$

The assumption $\lambda_{n} \leqslant 1 / 3$ for the Cantor sets $E(\lambda)$ is purely technical. Actually Theorem 1 (as well as Theorem 2 below) holds for any sequence $\left(\lambda_{n}\right)_{n}$ with $0<$ $\lambda_{n}<1 / 2$. See Remark 2] at the end of the paper for more details.

Mattila showed in [Ma] that the above condition is necessary and our contribution in this paper is to prove the sufficiency. The special case $\lambda_{n}=1 / 4, n \geqslant 1$, was obtained independently by Garnett [G1] and Ivanov [I1] in the 1970's and, since then, the "corner quarters" Cantor set has become the favorite example of a set of zero analytic capacity and positive length. P. Jones gave in [J] an alternative proof of Garnett's result, based on harmonic measure. Recently Jones' approach has been used to establish the vanishing of the analytic capacity of $E(\lambda)$ for some special classes of sequences $\lambda=\left(\lambda_{n}\right)_{n=1}^{\infty}$ with $4^{n} \sigma_{n}$ tending to infinity [GY].

Theorem 1 follows from a more precise result on the analytic capacity of the set $E_{N}=E\left(\lambda_{1}, \ldots, \lambda_{N}\right)$. The asymptotic behaviour of $\gamma^{+}\left(E_{N}\right)$ is completely 
understood: for some constant $C>1$ and all $N=1,2, \ldots$ one has

$$
C^{-1}\left(\sum_{n=1}^{N} \frac{1}{\left(4^{n} \sigma_{n}\right)^{2}}\right)^{-1 / 2} \leqslant \gamma^{+}\left(E_{N}\right) \leqslant C\left(\sum_{n=1}^{N} \frac{1}{\left(4^{n} \sigma_{n}\right)^{2}}\right)^{-1 / 2} .
$$

The upper estimate is due to Eiderman [E] and a different proof has been given in T2. The lower estimate was proved by Mattila in $\mathrm{Ma}$. However, the result was not explicitly stated in $\mathrm{Ma}$, presumably because at that time the main object of interest was $\gamma$ rather than $\gamma^{+}$. An indication of how one proves the lower estimate in (4) will be provided at the end of Section 2. See also [E, p. 821].

Theorem 1 follows from the upper estimate in (41) and the next result.

Theorem 2. There exists a positive constant $C_{0}$ such that

$$
\gamma\left(E_{N}\right) \leqslant C_{0} \gamma^{+}\left(E_{N}\right), N=1,2, \ldots
$$

If $\lambda_{n}=1 / 4, n \geqslant 1$, then combining Theorem 2 with (4), we get

$$
\gamma\left(E_{N}\right) \leqslant \frac{C}{\sqrt{N}}, N=1,2, \ldots,
$$

which improves considerably Murai's inequality [Mu

$$
\gamma\left(E_{N}\right) \leqslant \frac{C}{\log N}, N=2,3, \ldots,
$$

the best estimate known up to now.

The main tool used in our proof of Theorem 2 is the local $T(b)$-Theorem of M. Christ $\mathrm{CH} 2$, a particular version of which will be discussed and stated in Section 2. Section 2 also contains some basic facts on the Cauchy transform and the Plemelj formulae. The proof of Theorem 2 is presented in Section 3.

Our notation and terminology are standard. For example $D(z, r)$ is the open disk centered at $z$ and of radius $r, d s$ is the arclength measure on a rectifiable arc and $P \simeq Q$ means that $C^{-1} Q \leqslant P \leqslant C Q$ for some absolute constant $C>1$.

The symbols $C, C^{\prime}, C^{\prime \prime}, C_{0}, C_{1}, \ldots$ stand for absolute constants with a definite value. We will also use the symbol $A$ to denote an absolute constant that may vary at different occurrences.

Remark 1. The second author T3 has recently proved that Theorem 2 also holds for a general compact set. In particular, this implies the semi-additivity of analytic capacity. The proof in T3] also involves an induction argument and an appropriate $T(b)$-Theorem as in the present paper.

\section{BACKGROUND RESUlts}

2.1. Cauchy integrals. Fix an integer $M>0$ and let $E_{M}=E\left(\lambda_{1}, \ldots, \lambda_{M}\right)$ be the $M$-th approximation of the Cantor set associated with the sequence $\left(\lambda_{n}\right)_{n=1}^{\infty}$. Then $E_{M}$ is the union of $4^{M}$ closed squares $Q_{j}^{M}, 1 \leqslant j \leqslant 4^{M}$, and $\partial E_{M}$ is the union of the $4^{M}$ closed piecewise linear curves $\partial Q_{j}^{M}$. For a Borel measure $\mu$ supported on $\partial E_{M}$ set

$$
C(\mu)(z)=\int \frac{d \mu(\zeta)}{\zeta-z}, \quad z \notin \partial E_{M}
$$

and

$$
C(\mu)(z)=\lim _{\varepsilon \rightarrow 0} \int_{|\zeta-z|>\varepsilon} \frac{d \mu(\zeta)}{\zeta-z}, \quad z \in \partial E_{M}
$$


whenever the principal value integral exists. Let $C^{+}(\mu)(z)$ (respectively, $C^{-}(\mu)(z)$ ) stand for the non-tangential limit of $C(\mu)(w)$ as $w$ tends to $z$ from the interior of $E_{M}$ (respectively, from the complement of $E_{M}$ ). It follows from standard classical results that $C \mu(z), C^{+} \mu(z)$ and $C^{-} \mu(z)$ exist for almost all $z$ with respect to arclength measure $d s$ on $\partial E_{M}$. Moreover one has the Plemelj formulae (see [V3])

$$
\left\{\begin{array}{l}
C^{+} \mu(z)=C \mu(z)+\pi i f(z), \\
C^{-} \mu(z)=C \mu(z)-\pi i f(z),
\end{array}\right.
$$

where the identities hold for $d s$-almost all $z \in \partial E_{M}$ and $\mu=f(z) d z+\mu_{s}, f$ being integrable and $\mu_{s}$ being singular with respect to $d s$.

Assume that one has $\mu_{s}=0$ and

$$
|f(z)| \leqslant A, \text { for } d s \text {-almost all } z \in \partial E_{M},
$$

and that one wants to show

$$
|C(\mu)(z)| \leqslant A, \text { for } d s \text {-almost all } z \in \partial E_{M} .
$$

Then one only has to check that

$$
|C(\mu)(z)| \leqslant A, \text { for } z \notin E_{M},
$$

because then

$$
\left|C^{-}(\mu)(z)\right| \leqslant A, \text { for } d s \text {-almost all } z \in \partial E_{M},
$$

and thus the second identity in ([6) gives (17).

2.2. The local $T(b)$-Theorem. The local $T(b)$-Theorem is a criterion for the $L^{2}$ boundedness of a singular integral that was proved originally by $\mathrm{M}$. Christ in the setting of homogeneous spaces $\mathrm{CH} 2$. We state below a very particular version of Christ's result, which is adapted to the principal value Cauchy integral and to a measure $\mu$ supported on $\partial E_{M}$. The reader may think that $\mu$ is of the form $\mu=c d s_{\mid \partial E_{M}}$ for some (small) positive constant $c$. However, one should keep in mind that, for $4^{n} \sigma_{n} \nearrow \infty, d s_{\mid \partial E_{M}}$ does not satisfy condition (i) in the statement below with a constant independent of $M$. As will become clear later, an appropriate choice of $c$ is required to get (i) and (iii) with absolute constants.

Theorem (Christ). Let $\mu$ be a positive Borel measure supported on $\partial E_{M}$ satisfying, for some absolute constant $C$, the following conditions:

(i) $\mu(D(z, r)) \leqslant C r, z \in \partial E_{M}, r>0$.

(ii) $\mu(D(z, 2 r)) \leqslant C \mu(D(z, r)), z \in \partial E_{M}, r>0$.

(iii) For each disc $D$ centered at a point in $\partial E_{M}$ there exists a function $b_{D}$ in $L^{\infty}(\mu), b_{D}$ supported on $D$, satisfying $\left|b_{D}\right| \leqslant 1$ and $\left|C\left(b_{D} \mu\right)\right| \leqslant 1 \mu$-almost everywhere on $\partial E_{M}$, and $\mu(D) \leqslant C\left|\int b_{D} d \mu\right|$.

Then

$$
\int|C(f \mu)|^{2} d \mu \leqslant C^{\prime} \int|f|^{2} d \mu, \quad f \in L^{2}(\mu),
$$

for some absolute constant $C^{\prime}$ (depending only on $C$ ).

The relevance of inequality (8) for our problem lies in the fact that it implies

$$
\mu(K) \leqslant C^{\prime \prime} \gamma^{+}(K), \quad K \text { compact } \subset \partial E_{M},
$$

for some absolute constant $C^{\prime \prime}$ (depending only on $C^{\prime}$ ). 
The derivation of (9) from (8) goes through a well-known path: first, by classical Calderón-Zygmund theory one gets a weak $(1,1)$ inequality from (8); then, a surprisingly simple method to dualize a weak $(1,1)$ inequality leads immediately to (99). The original argument is in [DO]. Some years before [DO] Uy found a slightly different way of dualizing a weak $(1,1)$ inequality, which, however, does not yield (9) (see $|\mathrm{Uy}|)$. The interested reader will find additional information in [CH1], T1] and [V1].

Inequality (9) also explains why the lower estimate in (4) follows from Mattila's arguments in [Ma]; see Theorem 3.7 on p. 202 and the first paragraph after it.

\section{Proof of Theorem 2}

We first give a sketch of the argument. Assume that one can find a positive Borel measure $\mu$ supported on $\partial E_{N}, E_{N}=E\left(\lambda_{1}, \ldots, \lambda_{N}\right)$, which satisfies (i) and (ii) with $M$ replaced by $N$, such that $\|\mu\|=\gamma\left(E_{N}\right)$ and the Cauchy integral is bounded on $L^{2}(\mu)$. Then we get (9) with $M$ replaced by $N$, as we explained in Section 2. For $K=\partial E_{N}$ (9) yields

$$
\gamma\left(E_{N}\right)=\|\mu\| \leqslant A \gamma^{+}\left(E_{N}\right),
$$

as desired. In the actual argument we do not construct $\mu$ on $E_{N}$. For reasons that will become clear later, we are forced to work in $E_{M}$ with $M$ smaller than $N$. On the other hand, $M$ cannot be much smaller than $N$, because in the course of the subsequent reasoning one needs to have $\gamma^{+}\left(E_{M}\right) \leqslant A \gamma^{+}\left(E_{N}\right)$. Hence $M$ has to be chosen carefully, in such a way that the local $T(b)$-Theorem can be applied to get the boundedness of the Cauchy integral on $L^{2}(\mu)$, with an absolute constant.

Now we start the proof of Theorem 2.

Set $a_{n}=4^{n} \sigma_{n}$ and

$$
S_{n}=\frac{1}{a_{1}^{2}}+\frac{1}{a_{2}^{2}}+\cdots+\frac{1}{a_{n}^{2}} .
$$

We can assume, without loss of generality, that for each $N>1$, there exists $M$, $1 \leqslant M<N$, such that

$$
S_{M} \leqslant \frac{S_{N}}{2}<S_{M+1}
$$

Otherwise $\frac{S_{N}}{2}<S_{1}$ and thus, by (4),$\gamma^{+}\left(E_{N}\right) \geqslant A^{-1} \lambda_{1}$. On the other hand, taking into account the obvious estimate of analytic capacity by length, we clearly have

$$
\gamma\left(E_{N}\right) \leqslant \gamma\left(E_{1}\right) \leqslant \frac{1}{2 \pi} \operatorname{length}\left(\partial E_{1}\right)=\frac{8}{\pi} \lambda_{1} .
$$

Therefore (15) is trivial in the present case, provided $C_{0}$ is chosen to satisfy $C_{0} \geqslant \frac{8}{\pi} \mathrm{A}$.

Assume, then, that (10) holds and let us proceed to prove (5) by induction on $N$. The case $N=1$ is obviously true. The induction hypothesis is

$$
\gamma\left(E_{n}\right) \leqslant C_{0} \gamma^{+}\left(E_{n}\right), \quad 0<n<N,
$$

where the precise value of the absolute constant $C_{0}$ will be determined later.

We distinguish two cases.

Case 1: For some absolute constant $C_{1}$, to be determined later,

$$
a_{M} \gamma\left(E\left(\lambda_{M+1}, \ldots, \lambda_{N}\right)\right) \leqslant C_{1} \gamma\left(E_{N}\right) .
$$


Case 2: (11) does not hold.

We deal first with Case 2. By the induction hypothesis applied to the sequence $\lambda_{M+1}, \ldots, \lambda_{N}$ and by (4) we have

$$
\begin{aligned}
\gamma\left(E_{N}\right) & \leqslant C_{1}^{-1} a_{M} \gamma\left(E\left(\lambda_{M+1}, \ldots, \lambda_{N}\right)\right) \\
& \leqslant C_{1}^{-1} C_{0} a_{M} \gamma^{+}\left(E\left(\lambda_{M+1}, \ldots, \lambda_{N}\right)\right) \\
& \leqslant C_{1}^{-1} C_{0} A \frac{a_{M}}{\left(\sum_{n=M+1}^{N} \frac{1}{\left(4 \lambda_{M+1} \cdots 4 \lambda_{n}\right)^{2}}\right)^{1 / 2}} \\
& =C_{1}^{-1} C_{0} A \frac{1}{\left(\sum_{n=M+1}^{N} \frac{1}{a_{n}^{2}}\right)^{1 / 2}} .
\end{aligned}
$$

Clearly, the inequality $S_{M} \leqslant \frac{S_{N}}{2}$ is equivalent to

$$
\frac{1}{\left(\sum_{n=M+1}^{N} \frac{1}{a_{n}^{2}}\right)^{1 / 2}} \leqslant \frac{\sqrt{2}}{\left(\sum_{n=1}^{N} \frac{1}{a_{n}^{2}}\right)^{1 / 2}}
$$

and so, again by (4),

$$
\gamma\left(E_{N}\right) \leqslant C_{1}^{-1} C_{0} A \gamma^{+}\left(E_{N}\right)
$$

If $C_{1}=A$, where $A$ is the constant in the preceding inequality, we get (5), as desired.

Now let us now consider Case 1. Set

$$
\mu=\frac{\gamma\left(E_{N}\right)}{\operatorname{length}\left(\partial E_{M}\right)} d s_{\mid \partial E_{M}},
$$

so that $\|\mu\|=\gamma\left(E_{N}\right)$. To check condition (i) in the local $T(b)$-Theorem of Section 2 , we consider two cases. If $r \leqslant \sigma_{M}$, then

$$
\mu(D) \leqslant \frac{\gamma\left(E_{N}\right)}{\operatorname{length}\left(\partial E_{M}\right)} 2 r \leqslant C r
$$

because $\gamma\left(E_{N}\right) \leqslant \gamma\left(E_{M}\right) \leqslant \operatorname{length}\left(\partial E_{M}\right)$.

For $r>\sigma_{M}$ we can replace arbitrary discs centered at points in $\partial E_{M}$ by the squares $Q_{j}^{n}, 0 \leqslant n \leqslant M, 0 \leqslant j \leqslant 4^{n}$. In other words, it suffices to prove

$$
\mu\left(Q_{j}^{n}\right) \leqslant C \ell\left(Q_{j}^{n}\right), 0 \leqslant n \leqslant M, 1 \leqslant j \leqslant 4^{n} .
$$

To show this, given a disc $D$ of radius $r$ centered at $z \in \partial E_{M}$, one considers a square $Q_{j}^{n} \supset D$, where $n$ is chosen so that $\ell\left(Q_{j}^{n}\right)$ is comparable to $r$. Then,

$$
\mu\left(Q_{j}^{n}\right)=\gamma\left(E_{N}\right) \frac{1}{4^{n}}=\frac{4 \gamma\left(E_{N}\right)}{\operatorname{length}\left(\partial E_{n}\right)} \ell\left(Q_{j}^{n}\right) \leqslant \frac{4 \gamma\left(E_{n}\right)}{\operatorname{length}\left(\partial E_{n}\right)} \ell\left(Q_{j}^{n}\right) \leqslant \frac{2}{\pi} \ell\left(Q_{j}^{n}\right),
$$

and so (12) is proved.

It is also a simple matter to ascertain that (ii) holds. 
Now our goal is to prove that hypothesis (iii) of the local $T(b)$-Theorem is satisfied. Once this has been verified, (9) applied to $K=\partial E_{M}$ yields

$$
\gamma\left(E_{N}\right)=\|\mu\| \leqslant A \gamma^{+}\left(E_{M}\right) \leqslant \frac{A}{S_{M}^{1 / 2}} .
$$

Assuming that (13) holds, to complete the proof we again distinguish two cases, according to whether $1 / a_{M+1}^{2}$ is greater than $S_{M}$ or not.

If $1 / a_{M+1}^{2}>S_{M}$, then

$$
S_{M+1}=S_{M}+\frac{1}{a_{M+1}^{2}} \simeq \frac{1}{a_{M+1}^{2}}
$$

and so

$$
\gamma^{+}\left(E_{M+1}\right) \simeq \frac{1}{S_{M+1}^{1 / 2}} \simeq a_{M+1}=\frac{1}{4} \operatorname{length}\left(\partial E_{M+1}\right)
$$

Hence

$$
\gamma\left(E_{N}\right) \leqslant \gamma\left(E_{M+1}\right) \leqslant \frac{1}{2 \pi} \operatorname{length}\left(\partial E_{M+1}\right) \simeq \gamma^{+}\left(E_{M+1}\right) \simeq \gamma^{+}\left(E_{N}\right),
$$

and thus (5) holds for a sufficiently big constant $C_{0}$.

If $1 / a_{M+1}^{2} \leqslant S_{M}$, then $S_{M+1} \simeq S_{M}$ and so

$$
\gamma^{+}\left(E_{N}\right) \simeq \frac{1}{S_{N}^{1 / 2}} \simeq \frac{1}{S_{M}^{1 / 2}}
$$

which gives (5), with a big enough $C_{0}$, by (13).

Summing up, we have reduced the proof of Theorem 2 to checking that hypothesis (iii) of the local $T(b)$-Theorem is satisfied. As we already remarked when dealing with hypothesis (i), in proving (iii) we can replace discs centered at points in $\partial E_{M}$ by squares $Q_{j}^{n}, 1 \leqslant j \leqslant 4^{n}, 0 \leqslant n \leqslant M$. In other words, it is enough to show that, given a square $Q_{j}^{n}, 0 \leqslant n \leqslant M, 1 \leqslant j \leqslant 4^{n}$, there exists a function $b_{j}^{n}$ in $L^{\infty}(\mu)$, supported on $Q_{j}^{n}$, satisfying $\left|b_{j}^{n}\right| \leqslant 1$ and $\left|C\left(b_{j}^{n} \mu\right)\right| \leqslant 1 d \mu$-almost everywhere on $\partial E_{M}$ and such that

$$
\mu\left(Q_{j}^{n}\right) \leqslant C\left|\int b_{j}^{n} d \mu\right|
$$

Let $f$ be the Ahlfors function of $E_{N}$. Then $f$ is analytic on $\mathbb{C} \backslash E_{N},|f(z)| \leqslant 1$, $z \notin E_{N}, f(\infty)=0$ and $f^{\prime}(\infty)=\gamma\left(E_{N}\right)$. The non-tangential boundary value of $f$ at $\zeta \in \partial E_{N}$, which exists for $d s$-almost all $\zeta \in \partial E_{N}$, is denoted by $f(\zeta)$. Set $\nu=\frac{1}{2 \pi i} f(\zeta) d \zeta_{\mid \partial E_{N}}$, so that

$$
f(z)=\int \frac{1}{z-\zeta} d \nu(\zeta), \quad z \notin E_{N} .
$$

Fix a generation $n, 0 \leqslant n \leqslant M$. Then, for some index $k, 1 \leqslant k \leqslant 4^{n}$,

$$
\gamma\left(E_{N}\right)=\sum_{j=1}^{4^{n}} \nu\left(Q_{j}^{n}\right) \leqslant 4^{n}\left|\nu\left(Q_{k}^{n}\right)\right|,
$$

or, equivalently, $\mu\left(Q_{k}^{n}\right) \leqslant\left|\nu\left(Q_{k}^{n}\right)\right|$.

To define the function $b_{k}^{n}$ associated with $Q_{k}^{n}$, we need to describe a simple preliminary construction. 
Take a compactly supported $C^{\infty}$ function $\varphi$ on $\mathbb{C}, 0 \leqslant \varphi \leqslant 1, \int_{\partial Q^{0}} \varphi d s \geqslant 1$, such that $\varphi$ vanishes on $\bigcup_{j=1}^{4} D\left(z_{j}, 1 / 4\right)$, where the $z_{j}$ are the vertices of $Q^{0}$. Then $\left|C\left(\varphi d s_{\mid \partial Q_{0}}\right)\right| \leqslant A$, as one checks easily. Set

$$
\varphi_{j}^{M}(z)=\varphi\left(\frac{z-v_{j}^{M}}{\sigma_{M}}\right) \chi_{Q_{j}^{M}}
$$

where $v_{j}^{M}$ is the left lower vertex of $Q_{j}^{M}$. Hence $\left|C\left(\varphi_{j}^{M} d \mu\right)\right| \leqslant A$ and

$$
\int \varphi_{j}^{M} d \mu=\frac{1}{\operatorname{length}\left(\partial Q_{j}^{M}\right)} \int \varphi_{j}^{M} d s, \quad \mu\left(Q_{j}^{M}\right) \geqslant \frac{1}{4} \mu\left(Q_{j}^{M}\right) .
$$

Define

$$
b=b_{k}^{n}=\sum_{Q_{j}^{M} \subset Q_{k}^{n}} \nu\left(Q_{j}^{M}\right) \frac{\varphi_{j}^{M}}{\int \varphi_{j}^{M} d \mu} .
$$

For $j \neq k$ we construct $b_{j}^{n}$ by simply translating $b_{k}^{n}$. We have $Q_{j}^{n}=w_{j}^{n}+Q_{k}^{n}$, for some complex number $w_{j}^{n}$. Set

$$
b_{j}^{n}(z)=b_{k}^{n}\left(z-w_{j}^{n}\right), \quad z \in \mathbb{C} .
$$

Now we will prove that $b_{k}^{n}$ satisfies condition (iii). Clearly,

$$
\left|\int b d \mu\right|=\left|\nu\left(Q_{k}^{n}\right)\right| \geqslant \mu\left(Q_{k}^{n}\right)
$$

To show that $b$ is bounded, it suffices to prove

$$
\left|\nu\left(Q_{j}^{M}\right)\right| \leqslant A \mu\left(Q_{j}^{M}\right), \quad 1 \leqslant j \leqslant 4^{M}
$$

and for this we first remark that $\left|C\left(\chi_{Q_{j}^{M}} \nu\right)(z)\right| \leqslant A, z \notin E_{N}$. This is proved in [G2] Lemma 2.3 (a), p. 90]. Since $C\left(\chi_{Q_{j}^{M}} \nu\right)$ is analytic outside $Q_{j}^{M} \cap E_{N}$, we conclude that

$$
\left|\nu\left(Q_{j}^{M}\right)\right| \leqslant A \gamma\left(Q_{j}^{M} \cap E_{N}\right) .
$$

Now notice that the set $Q_{j}^{M} \cap E_{N}$ can be obtained from $E\left(\lambda_{M+1}, \ldots, \lambda_{N}\right)$ by a dilation of factor $\sigma_{M}$ and a translation. Hence, recalling (11),

$$
\gamma\left(Q_{j}^{M} \cap E_{N}\right)=\sigma_{M} \gamma\left(E\left(\lambda_{M+1}, \ldots, \lambda_{N}\right)\right) \leqslant C_{1} \frac{1}{4^{M}} \gamma\left(E_{N}\right)=C_{1} \mu\left(Q_{j}^{M}\right),
$$

which gives (14). It is worth noting at this point that the above inequality explains why $M$ cannot be taken to be $N$.

Thanks to the discussion in Section 2 on Cauchy integrals and the Plemelj formulae, it becomes clear that we are only left with the task of proving

$$
|C(b d \mu)(z)| \leqslant A, \quad z \notin E_{M} \cap Q_{k}^{n} .
$$

Since $\left|C\left(\chi_{Q_{k}^{n}} \nu\right)(z)\right| \leqslant A, z \notin E_{N} \cap Q_{k}^{n}$, we only need to estimate, for $z \notin E_{M} \cap Q_{k}^{n}$, the difference

$$
C(b d \mu)(z)-C\left(\chi_{Q_{k}^{n} \nu}\right)(z)=\sum_{Q_{j}^{M} \subset Q_{k}^{n}} C\left(\alpha_{j}^{M}\right)(z)
$$

where

$$
\alpha_{j}^{M}=\nu\left(Q_{j}^{M}\right) \frac{\varphi_{j}^{M} d \mu}{\int \varphi_{j}^{M} d \mu}-\chi_{Q_{j}^{M}} \nu
$$


We have $\int d \alpha_{j}^{M}=0$ and $\left|C\left(\alpha_{j}^{M}\right)(z)\right| \leqslant A, z \notin Q_{j}^{M}, 1 \leqslant j \leqslant 4^{M}$, again using [G2, Lemma 2.3 (a), p. 90].

Thus, if $z_{j}^{M}$ is the center of $Q_{j}^{M}$,

$$
\left|C\left(\alpha_{j}^{M}\right)(z)\right| \leqslant A \frac{\sigma_{M}^{2}}{\operatorname{dist}\left(z, Q_{j}^{M}\right)^{2}}, \quad\left|z-z_{j}^{M}\right|>\sigma_{M} .
$$

By the maximum principle, in estimating (15), we can assume that $\left|z-z_{j}^{M}\right| \leqslant \sigma_{M}$ for some $j$ with $Q_{j}^{M} \subset Q_{k}^{n}$. Hence (15) is not greater than

$$
A+A \sum_{l \neq j} \frac{\sigma_{M}^{2}}{\operatorname{dist}\left(z, Q_{l}^{M}\right)^{2}}
$$

For $0 \leqslant n \leqslant M$ let $Q^{n}$ be the square in the $n$-th generation that contains $Q_{j}^{M}$. We can estimate (177) by

$$
\begin{aligned}
& A+A \sum_{n=0}^{M-1} \sum_{Q_{l}^{M} \subset Q^{n} \backslash Q^{n+1}} \frac{\sigma_{M}^{2}}{\operatorname{dist}\left(z, Q_{l}^{M}\right)^{2}} \\
& \leqslant A+A \sum_{n=0}^{M-1} \frac{\sigma_{M}^{2}}{\sigma_{n}^{2}} 4^{M-n} \leqslant A+A \sum_{n=0}^{M-1}\left(\frac{4}{9}\right)^{M-n} \leqslant A,
\end{aligned}
$$

because $\sigma_{M}=\sigma_{n} \lambda_{n+1} \cdots \lambda_{M} \leqslant \frac{\sigma_{n}}{3^{M-n}}, 0 \leqslant n \leqslant M$.

This completes the construction of the function $b_{k}^{n}$ associated with the square $Q_{k}^{n}$ as required by hypothesis (iii) in the local $T(b)$-Theorem.

Now, by translation invariance it is clear that $b_{j}^{n}$ for $j \leqslant k$ also satisfies (iii).

This shows that the local $T(b)$-Theorem can be applied to $\mu$ and thus completes the proof of Theorem 2 .

Remark 2. For simplicity, we assumed above that $\lambda_{n} \leqslant 1 / 3$ for all $n$. However, both Theorem 1 and Theorem 2 hold for $0<\lambda_{n}<1 / 2$. Let us sketch the changes needed in the proof.

First it should be noticed that the estimate (44) for $\gamma^{+}\left(E_{N}\right)$ holds for $0<\lambda_{n}<$ $1 / 2$. Indeed, the arguments for the left inequality in (4) in [Ma are valid in this case. On the other hand, the right inequality in (4) is also true for $0<\lambda_{n}<1 / 2$. For example, arguing as in [T2], one can easily check that

$$
\gamma^{+}\left(E_{N}\right) \leqslant A\left(1+\sum_{\substack{1 \leqslant n \leqslant N, \lambda_{n} \leqslant 1 / 3}} \frac{1}{\left(4^{n} \sigma_{n}\right)^{2}}\right)^{-1 / 2} \approx\left(\sum_{1 \leqslant n \leqslant N} \frac{1}{\left(4^{n} \sigma_{n}\right)^{2}}\right)^{-1 / 2}
$$

The other places where the assumption $\lambda_{n} \leqslant 1 / 3$ has been used are (14) and (15). The inequality (14) also holds for $0<\lambda_{n}<1 / 2$. It follows from Vitushkin's estimates for the integral $\int_{\Gamma} f(z) d z$ for piecewise Lyapunov curves $\Gamma$ [Vi1] (in our case $\Gamma=\partial Q_{j}^{M}$ ). To prove (15), one can use the sharper estimate

$$
\left|C\left(\alpha_{j}^{M}\right)(z)\right| \leqslant A \frac{\sigma_{M} \gamma\left(Q_{j}^{M} \cap E_{N}\right)}{\operatorname{dist}\left(z, Q_{j}^{M}\right)^{2}} \leqslant A C_{1} \frac{\sigma_{M} \mu\left(Q_{j}^{M}\right)}{\operatorname{dist}\left(z, Q_{j}^{M}\right)^{2}}, \quad\left|z-z_{j}^{M}\right|>\sigma_{M},
$$

instead of (16) (see [G2, pp. 12-13], for example). We leave the details for the reader. 


\section{REFERENCES}

[CH1] M. ChRIST, "Lectures on singular integral operators", CBMS Regional Conference Series in Mathematics 77, American Mathematical Society, Providence, RI, 1990. MR 92f:42021

[CH2] M. Christ, A $T(b)$ theorem with remarks on analytic capacity and the Cauchy Integral, Colloq. Math. 60/61(2) (1990), 601-628. MR 92k:42020

[D] G. DAvid, Analytic capacity, Calderón-Zygmund operators, and rectifiability, Publ. Mat. 43(1) (1999), 3-25. MR 2000e:30044]

[DO] A. M. Davie, B. Øksendal, Analytic capacity and differentiability properties of finely harmonic functions, Acta. Math. 149 (1982), 127-152. MR 84h:31001

[E] V. Y. EIDERman, Hausdorff measure and capacity associated with Cauchy potentials, Math. Notes 63 (1998), 813-822. MR 99m:28015

[G1] J. Garnett, Positive length but zero analytic capacity, Proc. Amer. Math. Soc. 24 (1970), 696-699. MR 43:2203

[G2] J. Garnett, "Analytic capacity and measure", Lectures Notes in Mathematics 297, Springer-Verlag, Berlin-New York, 1972. MR 56:12257

[GY] J. Garnett, S. Yoshinobu, Large sets of zero analytic capacity, Proc. Amer. Math. Soc. 129 (2001), 3543-3548.

[I1] L. D. Ivanov, Variations of sets and functions, Nauka, Moscow, 1975 (Russian). MR 57:16498

[I2] L. D. Ivanov, On sets of analytic capacity zero, in "Linear and Complex Analysis Problem Book 3" (part II), Lectures Notes in Mathematics 1574, Springer-Verlag, Berlin, 1994, pp. 150-153.

[J] P. Jones, Square functions, Cauchy integrals, analytic capacity, and harmonic measure, in: "Harmonic Analysis and Partial Differential Equations", (El Escorial, 1987), Lectures Notes in Mathematics 1384, Springer, Berlin, 1989, pp. 24-68. MR 91b:42032

[Ma] P. Mattila, On the analytic capacity and curvature of some Cantor sets with non $\sigma$-finite length, Publ. Mat. 40 (1996) 195-204. MR 97d:30052

[Mu T. MuraI, Construction of $H^{1}$ functions concerning the estimate of analytic capacity, Bull. London Math. Soc. 19 (1987), 154-160. MR 88d:30034

[T1] X. Tolsa, $L^{2}$-boundedness of the Cauchy integral operator for continuous measures, Duke Math. J. 98(2) (1999), 269-304. MR 2000d:31001

[T2] X. TolsA, On the analytic capacity $\gamma^{+}$, Indiana Univ. Math. J. (51) (2) (2002), 317-344.

[T3] X. Tolsa, Painlevé's problem and the semiadditivity of analytic capacity, Acta Math. (to appear).

[Uy] N. X. Uy, Removable sets of analytic functions satisfying a Lipschitz condition, Ark. Mat. 17 (1979), 19-27.

[V1] J. Verdera, A weak type inequality for Cauchy transforms of finite measures, Publ. Mat. 36 (1992), 1029-1034. MR 94k:30099

[V2] J. VerderA, Removability, capacity and approximation, in: "Complex Potential Theory", (Montreal, PQ, 1993), NATO Adv. Sci. Int. Ser. C Math. Phys. Sci. 439, Kluwer Academic Publ., Dordrecht, 1994, pp. 419-473. MR 96b:30086

[V3] J. Verdera, $L^{2}$ boundedness of the Cauchy integral and Menger curvature, Contemporary Math. 277, American Mathematical Society, Providence, RI, 2001, pp. 139-158. MR 2002f:30052

[Vi1] A. G. Vitushin, Estimate of the Cauchy integral, Mat. Sb. 71(4) (1966), 515-534.

[Vi2] A. G. Vitushkin, Analytic capacity of sets in problems of approximation theory, Russian Math. Surveys 22 (1967), 139-200.

Departament de Matemàtiques, Universitat Autònoma de Barcelona, 08193 Bellaterra, (BARCElona), Spain

Département de Mathématiques, Université de Paris Sud 91405 Orsay, Cedex, France

Departament de Matemàtiques, Universitat Autònoma de Barcelona, 08193 Bellaterra, (BARCElona), Spain 